\title{
Density Based Traffic Control System with Smart Sensing Of Emergency Vehicles
}

\author{
Dr. M. Varadharaj \\ Associate professor-ECE, Kathir College of Engineering, Coimbatore, India \\ varatharajms80@gmail.com
}

\begin{abstract}
Present Traffic Light Controller (TLC) relies upon micro-controller and microchip. These TLC have restrictions as they are depend on pre-portrayed gear, which is filling in with respect to the program that doesn't have the versatility of adjustment on continuous reason. Owing to fixed time spans, orange and red signal's holding up time is more and vehicle uses more fuel. To make traffic light leadership progressively beneficial, we abuse the advancement of new procedure called as "Density based traffic control system with smart sensing of emergency vehicles". It is constructed mainly by using Magnetic Sensors for real world environment and by using IR modules for Model. The main objective of our project is to clear traffic efficiently by effective usage of the green signal time. In this system the density of the vehicle in a particular lane is obtained by the number of magnetic sensors kept in the road side which produces output signal with respect to the density of the traffic. Thus produced output signal is further processed by ARM microcontroller and according to the density obtained by the magnetic sensors the countdown time of the green signal is varied by the microcontroller and hence the usage of green signal even after all the vehicle pass by are prevented. In addition to this system our system also senses the emergency vehicle like ambulance that approaches the signal by detecting the RF signal transmitted by the Ambulance or other emergency vehicle with the help of $R F$ receivers that kept at the road side and halts all the vehicles by putting red signal for all the four sides of road and puts special 'green jeep signal' for the emergency vehicle to pass by hence our system provide way for emergency vehicle. It can also prioritize the emergency vehicle with the help of RF transmitter and receiver. As the signalling board receives the RF signal, it turns the Corresponding lane ON, thus clearing the route for the emergency vehicle. DSS also analyses the pollution levels by placing a check over the vehicle emissions at the junctions. When the priorities of any two lanes clash, pollution levels are taken into account to provide the signals for them in turns. The gas sensors are fitted onto the signalling boards which help in calculating the pollutant levels.
\end{abstract}

Keywords: Traffic light controller, ARM processor, Dynamic signaling, Emergency vehicles

\section{Introduction}

Traffic clog is a condition on street arranges that happens as use increments, and is described by more slow speeds, longer excursion times, and expanded vehicular lining. Clog has lead to delays, which may bring about late appearance for work, gatherings, and training, bringing about lost business, disciplinary activity or other individual misfortunes. Mileage on vehicles because of sitting in rush hour gridlock and successive speeding up and slowing down, prompts progressively visit fixes and substitutions. The squandered fuel builds air contamination and carbon dioxide outflows attributable to raising contamination levels. The Dynamic Signalling System (DSS) is an arrangement of equipment, programming, and administrators that permits better observing and control of traffic so as to streamline traffic stream. As a savvy arrangement toward enhancing traffic, DSS presents various advancements to diminish clog by checking traffic thickness using sensors, there by preempting the holding up time. The crisis vehicles are given specific gear, for example, RF transmitter, which permits crisis reaction vehicles to have high-need travel by having the lights along their course change to green. The vehicular outflow at each sign is observed so as to lessen the contamination levels in the district [1].

Vehicular Traffic control at street crossing focuses has dependably included worry for affiliations. A few endeavors have been made to structure productive motorized frameworks to deal with this issue. Most present 
day frameworks use pre-picked organizing circuits to work traffic signals. At any rate these structures are wasteful considering the way that they don't fill in as appeared by the existing quantity of traffic at the assembly. It is routinely found in the present mechanized busy time gridlock control structures that vehicles need to hold up at a street crossing despite the path that there is in every practical sense zero traffic the other way. There are different issues also, similar to ambulances getting made up for some ongoing difficulties by a red passage sign and expending huge time [2]. To deal with these issues and further upgrades to the marvellous issue of vehicular traffic direct at road crossing points we propose our endeavour "Thickness based traffic control system with quick identifying of emergency vehicles". Our idea relies upon the standard of obtaining thickness of vehicles with alluring sensors leading to rounds sides and moreover free course for savvy following from emergency vehicles [3].

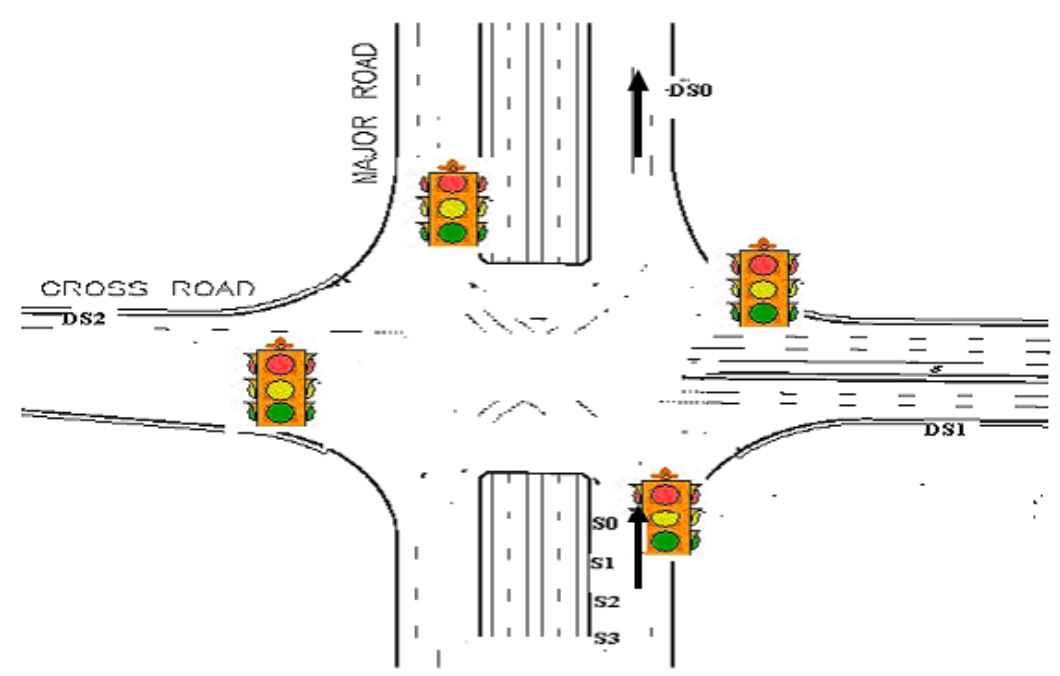

Fig 1: Basic Structure showing lane crossing for emergency vehicles

Existing System depends upon RFID. In this model two RFID per customers are put toward every method of a street crossing point and it has a focal PC structure to control. As a vehicle passes by a for each customer, it recovers its Electronic Product Code (EPC) information to the focal PC structure. The information got is then sent to the control station by remote or wired channels. The CDPS dealing with vehicular information and a DMS for controlling the traffic signals. The CDPS contains two zones. A mind boggling database where the records of vehicles before long passing the intersection point are immediately dealt with. A relentless database which has the records of all vehicles that crosses the intersection point.

The active database is partitioned into various parts. It masterminds the EPC information of vehicles as indicated by their way and course of movement. At whatever point a vehicle moves towards or away from the intersection, the two per users in its way distinguish it and pass on the acquired information to the CCS with some delay in the middle. The request for reaction of the two per users decides the course of movement of the vehicle. The vehicule information is then sent to any one piece of the database relating to its way and bearing of movement. What's more, programming and bolster faculty that are expected to introduce and work the RFID understanding frameworks (in a stockroom for instance) might be all the more exorbitant to utilize [4].

The primary impact of this issue is that expanded time squandering of the individuals out and about. The answer for this issue is by building up the program which distinctive setting delays for various intersections. No traffic, yet at the same time need to pause: At specific intersections, once in a while regardless of whether there is no traffic, individuals need to pause. The arrangement of this issue is by building up a framework which distinguishes traffic stream on every street and set timings of sign in like manner. Crisis vehicle stranded in car influx: Usually, during congested driving conditions, the crisis vehicle, for example, emergency vehicle, fire unit and police will be stuck particularly at the traffic light intersection. The arrangement is by utilizing RF Tx 
and Rx to square traffic on all the sides by applying red sign and by giving exceptional 'green jeep signal' for crisis vehicle to pass by [5].

\section{Existing techniques}

In our framework Magnetic sensors are utilized to gain the thickness of vehicles which are fixed inside a fixed separation. All the sensors are interfaced with microcontroller which thus controls the traffic signal framework dependent on the thickness identified by the sensors. On the off chance that the traffic is high on specific side more opportunity for green sign is designated for that side. The sensor ceaselessly continues detecting the thickness on all sides and the green sign clock is adjusted as needs be. In emergency vehicle and government vehicles RF transmitter is fixed and a RF collector is set half km away from signal. At the point when alarm is turned $\mathrm{ON}$, transmitter gets actuated and recipient kept at the street side recognizes the crisis vehicle signal. The discovery is sent as a message to the sign utilizing GSM innovation. When the message is gotten, RED light will show up on the remainder of the side streets and traffic will be cleared at the specific side [6].

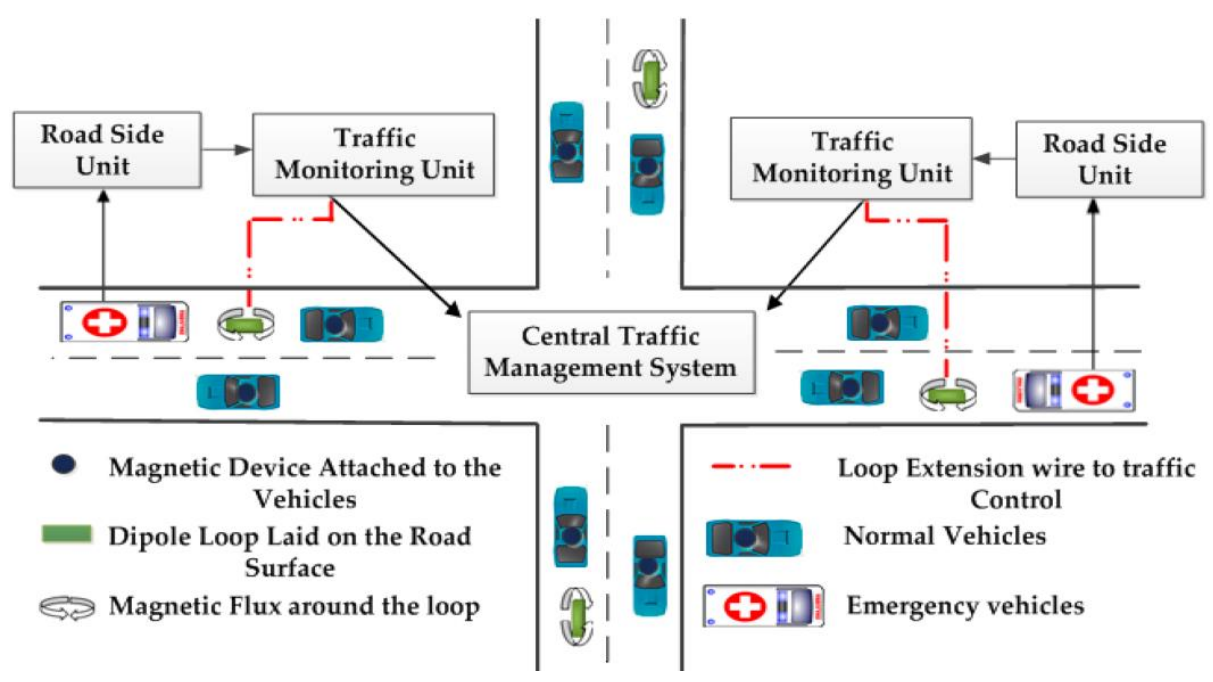

Fig2: Scenario showing existing lane crossing for emergency vehicles

The traffic light frameworks work on a planning instrument that changes the lights after a given peecharacterized stretch. It bombs in giving any sort of enhancement and stays fair-minded to the surpassing degree of traffic force. Our proposed framework is for the most part centred on setting sensors (S0, S1, S2, S3) along the paths for following the traffic thickness levels. In view of these dynamic readings, the sign gets pre-empted. The framework likewise targets giving snappier access to the courses for the crisis vehicles by shutting the traffic on the various paths. Furthermore, DSS screens the vehicular discharges along these lines advancing the contamination at the sign intersections.

The PIC Micro-controller board comprises of circuits important to work a Micro-controller with PC interface. The board contains arrangements for interfacing 8 simple information sources and 23 Digital level signs. It has high memory limit that broadens up to 368 x 8 bytes of Data Memory (RAM), 256 x 8 bytes of EEPROM Data Memory and 8K x 14 expressions of FLASH Program Memory [7]. The IR Emitter continually radiates 5V to the finder which is thus given to the miniaturized scale controller. As the beam gets obstructed, the gracefully is pulled back from the controller, which hint the arrangement of the traffic thickness.

The RF transmitter recipient the sign from the miniaturized scale controller and it is transmitted to the optional area. For example to the RF collector which gets the sign from the RF transmitter and sends it to the small scale controller present in the optional segment. A versatile traffic control framework was created where the traffic load is persistently estimated by sensors associated with a PIC miniaturized scale controller, based framework. The nearness of the vehicle is identified at whatever point the sign from the IR Transmitter to the IR Receiver 
gets blocked[8][9]. To help crisis vehicles, the ordinary tasks of traffic lights are pre-empted, utilizing Signal Pre-emption. The most widely recognized utilization of these frameworks is to control traffic signals in the way of a crisis vehicle utilizing RF transmitter and recipient, halting clashing traffic and permitting the crisis vehicle option to proceed, to help lessen reaction times and improve traffic security. The path wherein the crisis vehicle is distinguished, is constantly given the top most need, along these lines making the way course. The gas sensors are utilized to recognize the degree of emanation depleted from different vehicles at the sign intersection and send the incentive to the smaller scale controller. The smaller scale controller contrasts the deliberate worth and the given indicated esteem which thus relies upon the traffic power. At whatever point the thickness of two streets is same, the sign pre-emption is done dependent fair and square of toxins from the vehicles [10].

\section{Proposed Methodology}

Dynamic Signalling System (DSS) functionalities are controlled by the ARM microcontroller which acts as the governing agent. Through the help of IR sensors, the lane density is identified by the microcontroller which in turn controls the signalling board pre-emption. The density thus calculated is used to allocate adequate seconds to each lane to get through the traffic. For each turn, the readings change dynamically based on the traffic that prevails. The flow graph of the proposed methodology is shown in figure 3 .

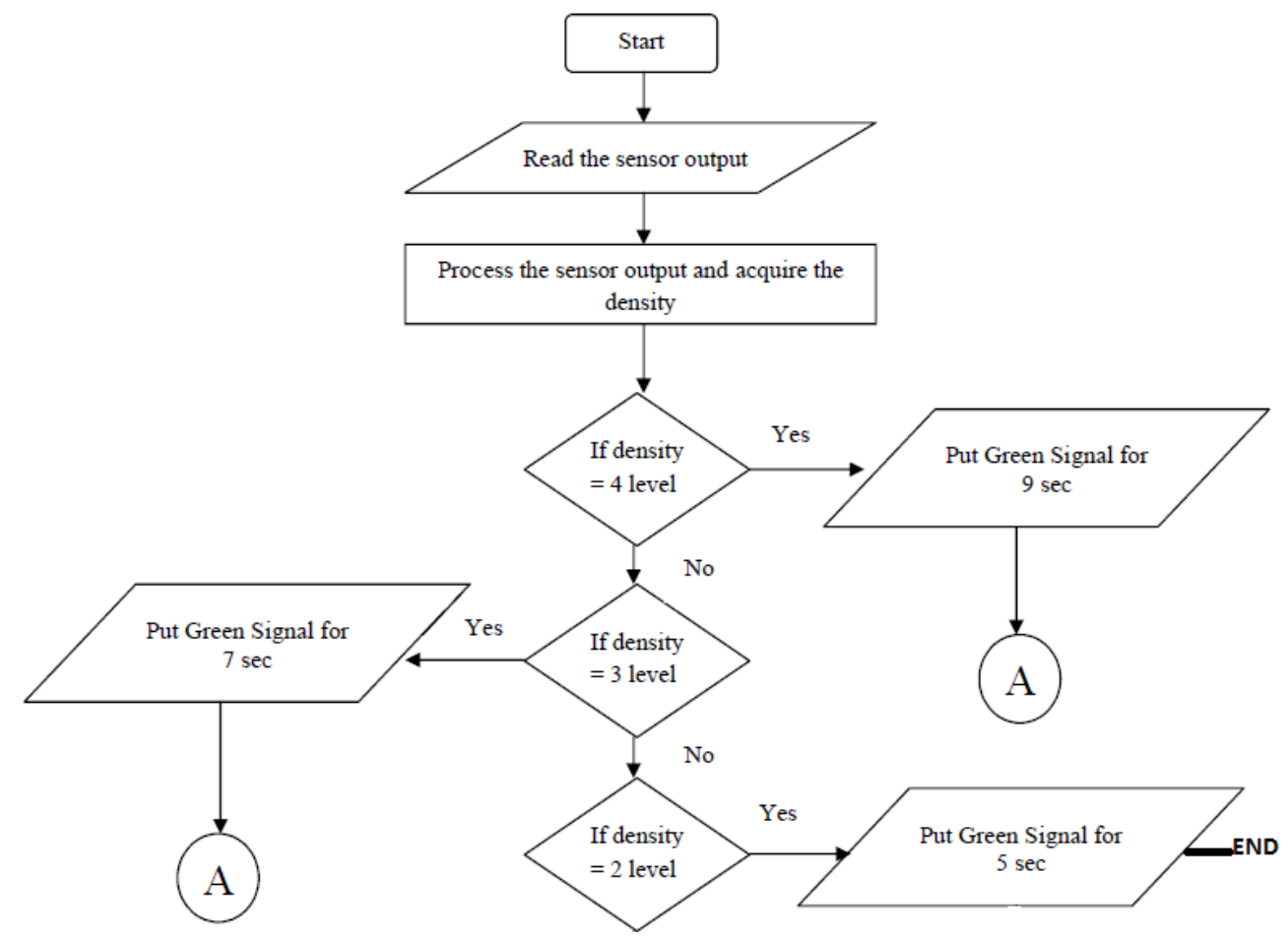

Fig3: Flow graph showing proposed lane crossing for emergency vehicles

Each emergency vehicle is equipped with RF transmitter to indicate its presence on the particular lane. The RF signals emitted by the emergency vehicles are captured by the RF receiver and are presented to the microcontroller based on which the signal pre-emption is performed. The gas sensors are placed on the signal conditioning board which constantly monitors the level of emission exhaust contained in the air. This input is taken into account whenever the density of any two lanes remains the same. The signalling board accommodates to the dynamic nature of the increasing traffic through these various inputs.

The varying levels of traffic intensity need a well established signalling system that constantly monitors the presence of vehicles along the lane, which is provided through our project. DSS also provides a solution for the 
emergency vehicles to evade the traffic congestion. It also extends its service towards prioritizing the emergency vehicles and by constantly monitoring the pollutants in the air. If our project gets implemented, it would carve a niche for itself in the IEEE-Intelligent Traffic System (ITS).

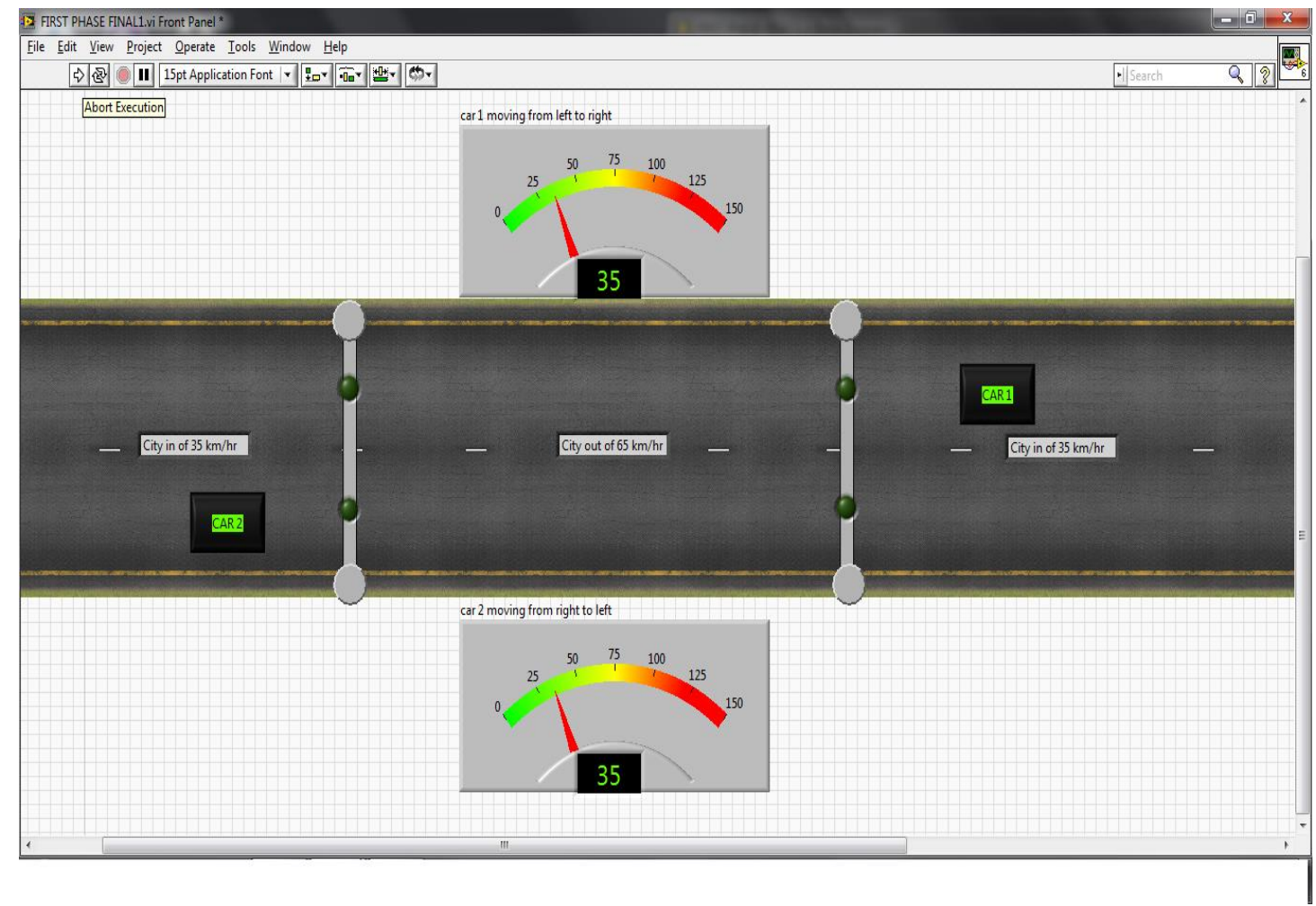

Fig 4: Structure of the proposed system

In urban areas, the traffic is ending up being dead significant issue and tries are taken to pick the traffic issues. In the proposed system, the traffic light posts are at the motivation behind association of traffic crossing point which is compelled by the little degree controller endless nimbly of vehicles moving closer at the get together. The vehicles are outfitted with RFID tag and the stand-separated tag and the vehicle owner's information will be chosen at the central database. The mix has a RFID per client that takes a gander at the names moving nearer towards the convergence point. Subordinate upon the proportion of vehicles read by the RFID per client, the cut back scale controller will set the time and control the sign as demonstrated by the check. The emergency vehicles are furnished with an astounding conspicuous evidence tag. The emergency vehicles like salvage vehicle, fire parcel, police vehicles and VIP vehicles will have novel RFID marks when such vehicles come at the traffic crossing point the RFID per client explores these remarkable names and control unequivocal course sign to green in this manner allowing the emergency vehicles go viably through the mix and in this way keeping up a basic not too bad ways from wastage of time.

\section{Simulation Results}

Traffic light posts are organized at the traffic gathering. Traffic light set the green portion for unequivocal time range which is by no means whatsoever, a firm precise structure as it can't deal with the traffic issues totally. The proposed structure will have RFID per clients at the traffic congregations that will take a gander at all the RFID marks joined to the vehicles coming towards the crossing point. Right when the crisis vehicle goes for the salvage vehicle to reach to within through subsequently clearing the traffic and before it shows up at the traffic signal. The third part is for attestation of taken vehicle. Here, when the RFID per client takes a gander at the RFID tag, it considers it to the picked taken RFIDs. Besides GPS can be set close to the taken vehicle 
certification module, so the particular region of taken vehicle is known. In case a match is found, it sends SMS to the police control room, with the objective that the vehicle is made to stop in the dynamic time gridlock crossing point and close to police can make fitting move.

This proposed system has three domains. Starting territory contains changed sign control structure. Here, each vehicle is outfitted with a RFID tag and per client at traffic crossing point. Exactly when it comes in the level of RFID per client, it will offer the hint to the RFID per client which will follow what number of vehicles has experienced the convergence point for a specific time range and pick the traffic stop up volume. In like way, it sets the green zone for that particular way. Second part is for the emergency vehicle district and room. Here, each emergency vehicle contains Zig-Bee transmitter and the Zig-Bee beneficiary will be open at the traffic crossing point. The cost will be turned $\mathrm{ON}$ when the vehicle is used for emergency.

This will introduce the sign through the Zig-Bee transmitter to the discoverer. It will turn the traffic light to change to green. Precisely when the crisis vehicle experiences gathering, the position no longer gets the Zig Bee signal and the traffic light is changed to red. RFID and GPS based changed traffic breathing space structure for crisis vehicle. The motivation behind association of this work is to decrease the deferral for the crisis vehicle to reach to the clinical office by techniques for consistently making the traffic way, before it shows up at the traffic signal. The third part is for revelation of taken vehicle. Here, when the RFID per client examines the RFID tag, it takes a gander at it to the chose taken RFIDs. Moreover GPS can be set close to the taken vehicle indisputable affirmation module, so the particular locale of taken vehicle is known. If a match is found, it sends SMS to the police control room, with the objective that the vehicle is made to stop in the dynamic time gridlock crossing point.

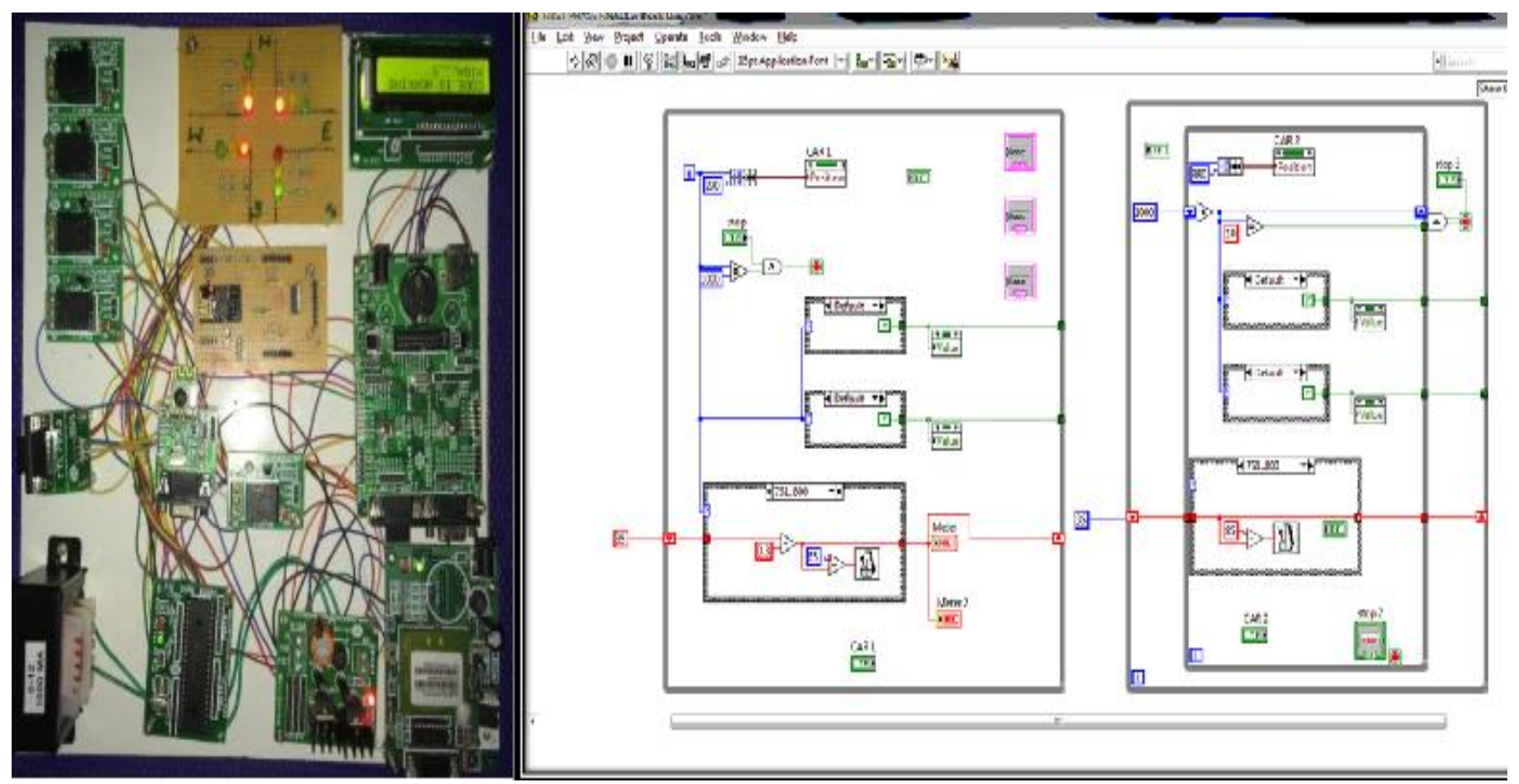

Fig 5: Hardware setup of the proposed system with Lab-view panel diagram

The past frameworks work productively to figure out how to deal with the traffic yet doesn't give nature of administration to crisis and taken vehicles and utilize extra equipment like Zig-Bee and so on and muddle the framework. The proposed framework works successfully and with higher exactness in dealing with the traffic at intersection with various needs at various times. RFID along with Internet and GSM advances are foreseen to make a transformation in rush hour gridlock the board and control frameworks. The information base contains online measurable data, which can be utilized by administrators and organizers to grow better models later on. The framework spares important subtleties in the records of the database, which can give significant data to organizers and examiners. Further upgrades should be possible to the model by testing it with GPS, so the 
specific area of taken vehicle is known. It very well may be improved by reaching out in a multi-street intersection.

\section{Conclusion}

Thus the "Density Based Traffic Control System with Smart Sensing of Emergency Vehicles" has been simulated with the help of Lab-view simulation software by using switches instead of magnetic sensors in order to acquire density of the traffic and the green signal time is controlled with respect to the density obtained by the switches. Hence the desired output is obtained. In future our project will be extended to control all the four lanes in a signal. This is made possible by interfacing all the four lanes to a single controller. Interfacing will be done efficiently using PIC interfacing techniques. In addition to this Emergency and government vehicle detection is made possible by RF transmitter and RF receiver collectively known as RF transceiver.

\section{References}

[1] Cascetta, E., Inaudi, D., and Marquis, G., "Dynamic estimators of origin-destination matrices using traffic counts", Transportation Science, Vol. 27, Issue 4, 1993, pp. 363-373.

[2] W. Wen, "A dynamic and automatic traffic light control system for solving the road congestion problem”, Expert Systems with Applications, Vol. 34, Issue 4, May 2008, pp. 2370-2381.

[3] Yi Hu, Peter Thomas, and Russel J. Stonier, "Traffic signal control using fuzzy logic and evolutionary algorithms”, IEEE Congress on Evolutionary Computation, 2007, pp.1785-1792.

[4] Chattaraj, S. Chakrabarti, S. Bansal, S. Halder, A. Chandra, "An Intelligent Traffic Control System using RFID",IEEE Potentials, vol. 28, no. 3, pp. 40-43, May-Jun. 2009.

[5] R. Hegde, R. R. Sali, M. S. Indira, "RFID and GPS based automatic lane clearance system for ambulance",Int. J. Adv. Elect. Electron.Eng., vol. 2, no. 3, pp. 102-107, 2013.

[6] J.Sherly,D.Somasundareswari "INTERNET OF THINGS BASED SMART TRANSPORTATION SYSTEMS" International Research Journal of Engineering and Technology (IRJET)2015.Ayoub Fikri,El Mehdi Zrihni,YassineSalihAlj ”A Smartphone Based System for Traffic Congestion Control using RFID tags" International Conference on Electrical and Information Technology ICEIT2015

[7] N. Rida, M. Ouadoud, A. Hasbi and S. Chebli, "Adaptive Traffic Light Control System Using Wireless Sensors Networks," 2018 IEEE 5th International Congress on Information Science and Technology (CiSt), Marrakech, 2018, pp. 552-556, doi: 10.1109/CIST.2018.8596620.

[8] Sreemana Datta, "Real time road traffic light signaling using image processing with emergency vehicle detection and traffic signal violation detection", Scholars Journal of Engineering and Technology, 2013.

[9] X. Wen-juan and L. Jian-feng, "Application of vision sensing technology in urban intelligent traffic control system," 2018 4th International Conference on Computer and Technology Applications (ICCTA), Istanbul, 2018, pp. 74-77, doi: 10.1109/CATA.2018.8398659.

[10] C. Ma, W. Hao, A. Wang and H. Zhao, "Developing a Coordinated Signal Control System for Urban Ring Road Under the Vehicle-Infrastructure Connected Environment," in IEEE Access, vol. 6, pp. 52471-52478, 2018, doi: 10.1109/ACCESS.2018.2869890. 